\title{
Electrically tunable transverse magnetic focusing in graphene
}

\author{
Thiti Taychatanapat ${ }^{1,2}$, Kenji Watanabe ${ }^{3}$, Takashi Taniguchi ${ }^{3}$ and Pablo Jarillo-Herrero ${ }^{2 \star}$
}

\begin{abstract}
Electrons in a periodic lattice can propagate without scattering for macroscopic distances despite the presence of the nonuniform Coulomb potential due to the nuclei'. Such ballistic motion of electrons allows the use of a transverse magnetic field to focus electrons ${ }^{2}$. This phenomenon, known as transverse magnetic focusing (TMF), has been used to study the Fermi surface of metals $^{3}$ and semiconductor heterostructures ${ }^{4}$, as well as to investigate Andreev reflection ${ }^{3}$ and spin-orbit interaction ${ }^{5}$, and to detect composite fermions $s^{6,7}$. Here we report on the experimental observation of TMF in high-mobility mono-, biand tri-layer graphene devices. The ability to tune the graphene carrier density enables us to investigate TMF continuously from the hole to the electron regime and analyse the resulting focusing fan. Moreover, by applying a transverse electric field to tri-layer graphene, we use TMF as a ballistic electron spectroscopy method to investigate controlled changes in the electronic structure of a material. Finally, we demonstrate that TMF survives in graphene up to $300 \mathrm{~K}$, by far the highest temperature reported for any system, opening the door to new room-temperature applications based on electron-optics.
\end{abstract}

The concept of TMF can be illustrated by considering electrons entering a two-dimensional system through a narrow injector (origin in Fig. 1a). In the presence of a magnetic field $B$, electrons will undergo cyclotron motion with radius $r_{\mathrm{c}}$ and get focused on the caustic (a quarter of a circle with radius $2 r_{\mathrm{c}}$ ) on which the electron density becomes singular (Fig. 1a, top). Moreover, the specular reflection of electrons at the boundary of the two-dimensional system causes a skipping orbit motion that results in focal points at integer multiples of $2 r_{\mathrm{c}}$ along the $x$ axis (Fig. 1a, bottom). This basic behaviour still holds for electron motion in a solid as long as the Fermi surface has cylindrical symmetry ${ }^{3}$. Hence, the magnetic field, $B_{\mathrm{f}}$, required to focus electrons at a distance $L$ is

$$
B_{\mathrm{f}}^{(p)}=\left(\frac{2 \hbar k_{\mathrm{F}}}{e L}\right) p=\left(\frac{2 \hbar \sqrt{\pi n}}{e L}\right) p
$$

where $p-1$ is the number of reflections off the edge of the system (for example, $p=1$ corresponds to a direct injector to collector trajectory, without reflections), $\hbar$ is the reduced Planck constant, $e$ is the elementary charge and $k_{\mathrm{F}}=\sqrt{4 \pi n / g_{\mathrm{s}} g_{\mathrm{v}}}$ is the Fermi momentum, with $n$ being the carrier density and $g_{\mathrm{s}}=g_{\mathrm{v}}=2$ being spin and valley degeneracies respectively. For negative density, the direction of $B_{\mathrm{f}}$ has to be reversed as the carrier charge changes sign (Fig. 1b).

To study TMF in graphene, we fabricate Hall bar devices based on high-mobility mono- (MLG), bi- (BLG) and tri-layer (TLG) graphene on hexagonal boron nitride (hBN) substrates ${ }^{8-10}$ (see Methods and Fig. 1c). Figure 1d shows the resistivity of a
MLG device as a function of density at zero magnetic field. Its field-effect mobility is $\sim 100,000 \mathrm{~cm}^{2} \mathrm{~V}^{-1} \mathrm{~s}^{-1}$ at low temperature, corresponding to a mean free path of $\sim 1 \mu \mathrm{m}$. A similar behaviour is observed for BLG and TLG devices. The high mobility and low disorder enable us to probe TMF in these devices.

We employ the measurement configuration shown in Fig. 1c to probe the focusing of electrons (see Methods). Figure 1e shows the collector voltage $V_{\mathrm{c}}(B, n)$ normalized by the injected current $I_{\mathrm{i}}$ in MLG, at $5 \mathrm{~K}$. For $|B| \geq 2.5 \mathrm{~T}$, we observe Shubnikov-de Haas oscillations (SdHOs), as expected from a generalized longitudinal resistance measurement. However, the SdHOs are nearly invisible in quadrants 1 and 3, owing to the interference of different trajectories of electrons propagating coherently to the collector ${ }^{4,11-13}$ (see Supplementary Information).

For $|B| \leq 2.5 \mathrm{~T}$, we observe three unusual peaks that do not resemble SdHOs. The location of these peaks in the $B-n$ plane (see equation (1)) indicates that they correspond to TMF. The peaks arise when electrons are focused onto the collector, resulting in a build-up of $V_{\mathrm{c}}$. The first peak corresponds to electrons propagating directly from the injector to the collector whereas for the subsequent peaks electrons reflect off the edge before reaching the collector (Fig. 1e, top insets). For positive density, positive $B$ directs the charge carriers to the collector whereas negative $B$ forces them to propagate away, resulting in no focusing peak (Fig. 1e, top insets). For negative density, the sign of the charge carriers flips, and therefore $B$ has to be reversed to focus the carriers at the collector. The ability to tune carrier density in graphene enables us to investigate the $\sqrt{n}$ dependence of the focusing fields, or focusing fan, continuously from the electron to hole regimes.

The values of $B_{\mathrm{f}}$ can be readily calculated, because both $n$ and $L$ can be obtained from Hall and atomic force microscopy (AFM) measurements, respectively. Figure 2 a shows a zoom-in plot of Fig. 1e, where we have superimposed the calculated focusing fields (dashed lines) using the measured $L_{\mathrm{MLG}}=500 \mathrm{~nm}$. A discrepancy between the calculated values and the measured peak locations is clearly present. Moreover, we find that the observed $B_{\mathrm{f}}^{(p)} / p$ decreases as $p$ increases (see Supplementary Information). The finite width of our injector and collector $(\sim 100 \mathrm{~nm}$ in MLG and BLG and $\sim 240 \mathrm{~nm}$ in TLG, see below) could introduce an error in $L$ and subsequently $B_{\mathrm{f}}$. However, a more plausible explanation is the effect of charge accumulation near the edges owing to the finite size of our graphene devices ${ }^{14}$. We find that charge accumulation reduces the values of $B_{\mathrm{f}}$. It also explains the decreasing $B_{\mathrm{f}}^{(p)} / p$ because, for higher $p$, the carrier's trajectory is closer to the edge, which further reduces $B_{\mathrm{f}}^{(p)}$ (see Supplementary Information). The effect of spatially varying density on $B_{\mathrm{f}}$ has also been discussed in heterostructures as a result of their soft-wall potential ${ }^{7}$. Density fluctuations and small-angle

${ }^{1}$ Department of Physics, Harvard University, Cambridge, Massachusetts 02138, USA, ${ }^{2}$ Department of Physics, Massachusetts Institute of Technology, Cambridge, Massachusetts 02139, USA, ${ }^{3}$ National Institute for Materials Science, Namiki 1-1, Tsukuba, Ibaraki 305-0044, Japan. *e-mail: pjarillo@mit.edu. 


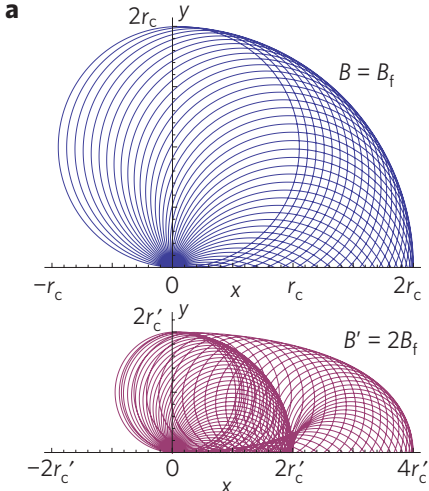

b

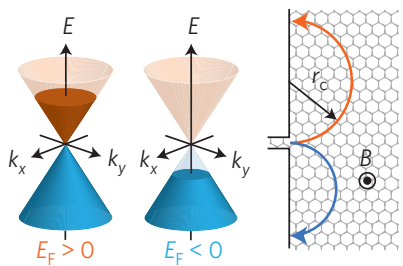

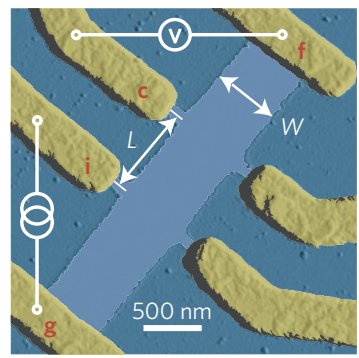

d

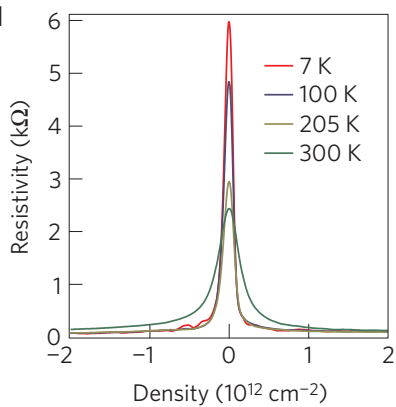

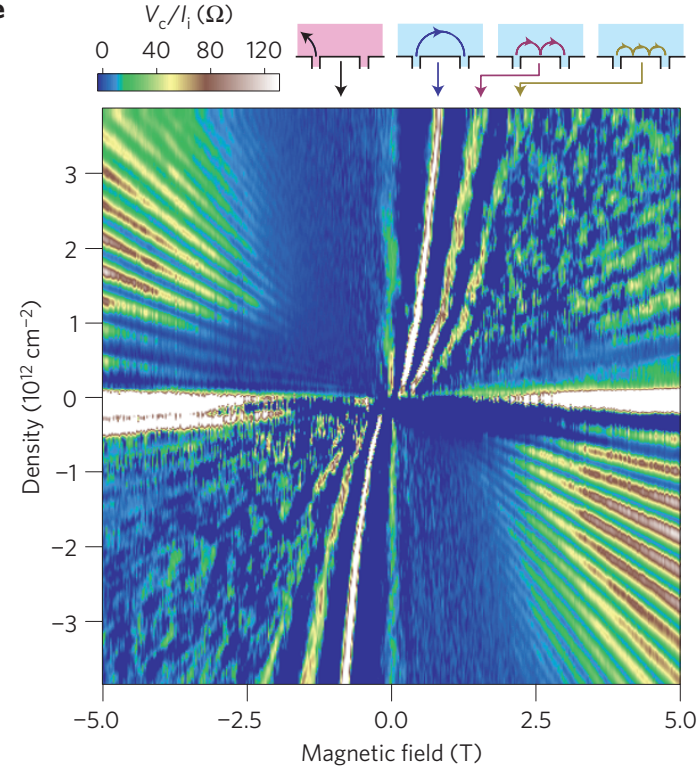

Figure 1 | TMF schematics. a, Classical trajectories of electrons injected isotropically from the origin at $B=B_{\boldsymbol{f}}$ (top) and $B^{\prime}=2 B_{\mathfrak{f}}$ (bottom, including one bounce off the edge). Electrons are focused at an integer multiple of $2 r_{c}$ along the $x$ axis. $\mathbf{b}$, Schematics depicting the band structure of MLG at positive (left) and negative (centre) electron density. Electron's trajectories at a finite $B$ are shown on the right for Fermi energy $E_{F}>0$ (orange line) and $E_{F}<0$ (blue line). c, False-colour AFM image of a TMF device. In the TMF measurement, contact i injects current $l_{\mathrm{i}}$ into graphene and the voltage $V_{\mathrm{c}}$ is measured at the collector (contact c) relative to contact f. $L$ is the measured distance between the centres of contacts $i$ and c. d, Resistance versus gate voltage of MLG at various temperatures measured in the usual 4-probe Hall-bar geometry. e, TMF spectrum in MLG at $5 \mathrm{~K}$. TMF peaks from first, second and third modes can be observed clearly for $|B|<2.5 \mathrm{~T}$. The top insets show representative trajectories for each corresponding mode. At higher $B$, SdHOs are also present.

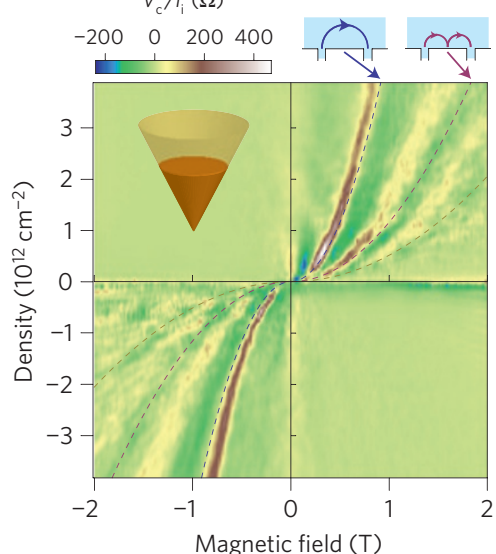

b

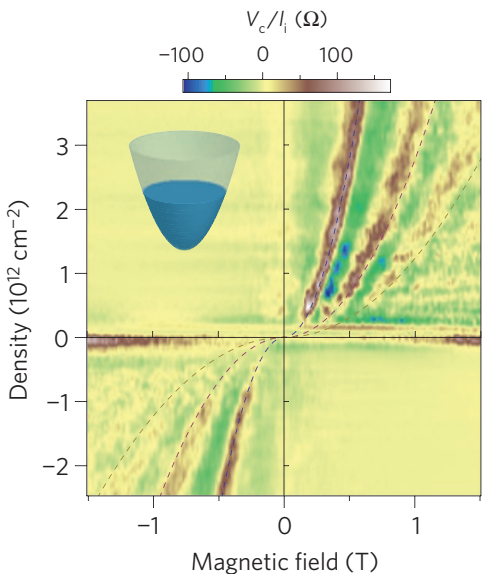

c

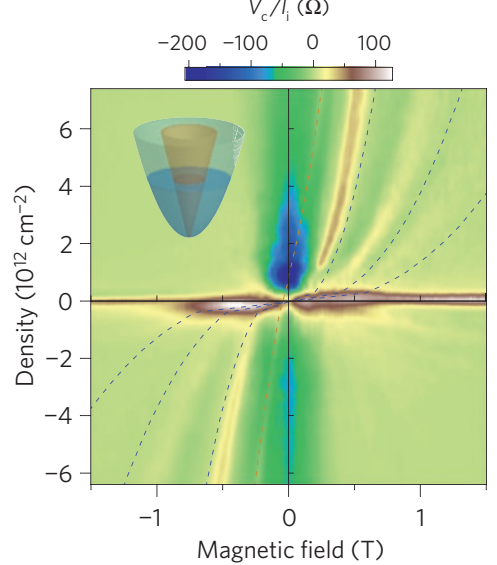

Figure 2 | TMF in MLG, BLG and TLG at 5 K. a-c, The TMF spectra as a function of density and magnetic field for MLG (a), BLG (b) and TLG (c). The dashed lines are calculated focusing fields using $L_{M L G}=500, L_{B L G}=775$ and $L_{T L G}=950 \mathrm{~nm}$, determined from AFM images. In $\mathbf{c}$, the orange and blue lines are the calculated focusing fields from MLG-like and BLG-like subbands respectively. Insets are the band structures when only $\gamma_{0}$ and $\gamma_{1}$ are taken into account.

scattering ${ }^{12}$ due to impurities could also affect the carrier's path and $B_{\mathrm{f}}$ but a lack of the detailed potential landscape prevents us from performing quantitative analysis.

We have observed multiple focusing peaks in all of our devices, including BLG and TLG (see below), which indicates that a significant fraction of the electrons get specularly reflected off the graphene edge. The measured specularity, the ratio between the amplitudes of the second and first peaks, offers information on the specular reflection of electrons at the graphene edges. We find that the value of specularity ranges from 0.2 to 0.5 (see Supplementary Information). It is worth noting that specularity measurements in semiconductor heterostructures have shown values less than 1 for focused-ion-beam-etched devices, which is similar to our oxygen-plasma-etched graphene devices but greater than 1 for electrostatically defined edges (see ref. 3 and references therein).

Similar behaviour is also observed in BLG and TLG. Figure $2 \mathrm{a}-\mathrm{c}$ shows the focusing fans at $5 \mathrm{~K}$ for MLG, BLG and TLG, respectively. Evidently, the TMF spectra of MLG and BLG are very similar, even though their band structures are different (Fig. 2a,b, insets). The similarity arises from the fact that, when only the nearest intra-layer $\gamma_{0}$ and inter-layer $\gamma_{1}$ hopping parameters are considered, both MLG and BLG have circular Fermi surfaces, resulting in the same circular orbit and $\sqrt{n}$ dependence of $k_{\mathrm{F}}$. The dashed lines in Fig. $2 \mathrm{~b}$ are focusing fields calculated from equation (1) which show a discrepancy, similar to the MLG case. Another possible source of mismatch in BLG, which does not exist in MLG, is the trigonal warping effect ${ }^{15}$, which transforms the BLG circular Fermi surface 

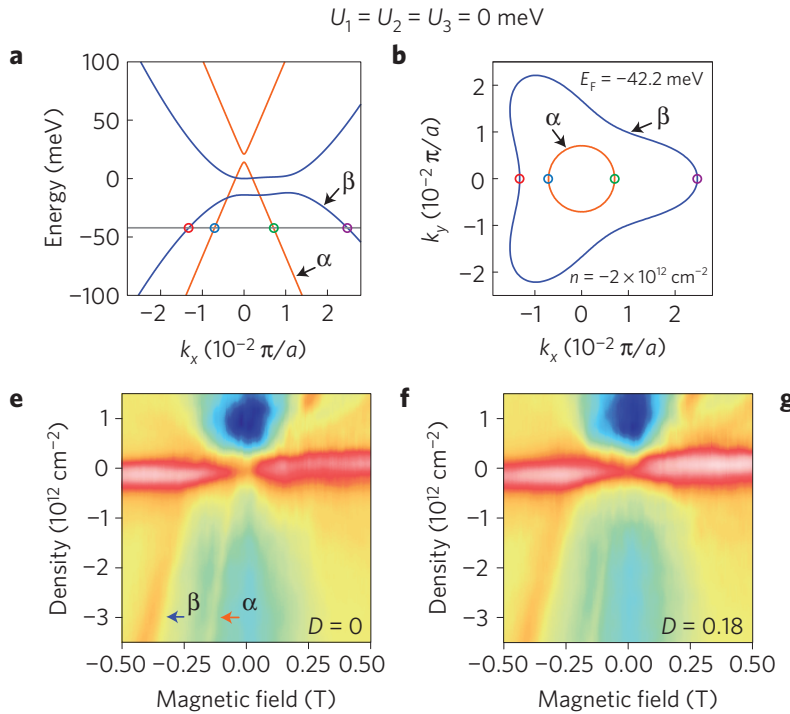

$U_{1}=-30, U_{2}=0, U_{3}=30 \mathrm{meV}$
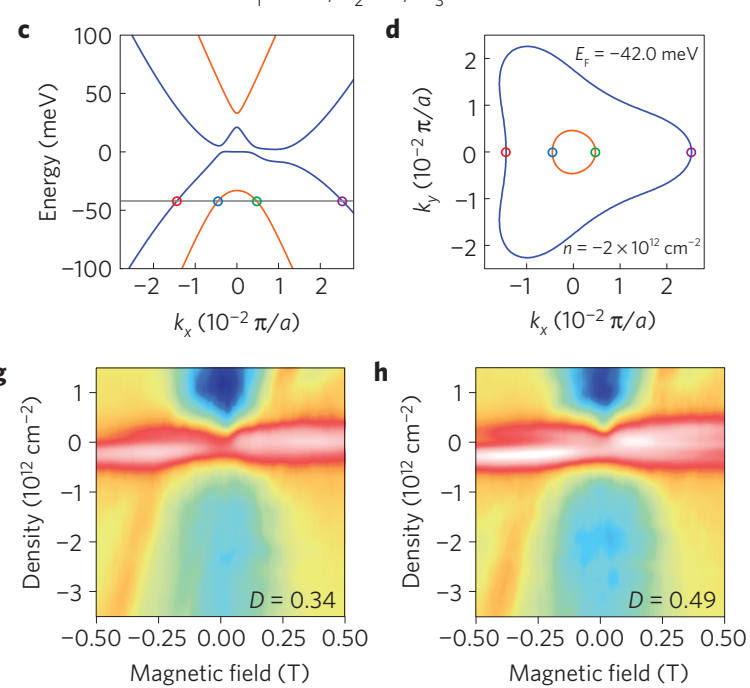

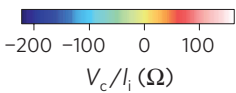

Figure 3 | Visualizing in situ band structure changes in TLG with TMF. a,b, Band structure and Fermi surface of TLG at zero $D$ (electrostatic potential of each layer equal to zero). The band structure consists of MLG-like and BLG-like subbands, with a small band overlap. The bands $\alpha$ and $\beta$ are MLG-like and BLG-like valence bands. The trigonal warping effect can be seen in the BLG-like subband. The lattice constant $a$ is $2.46 \AA$. c,d, Band structure and Fermi surface of TLG at finite $D$ (for this case, with potential difference between adjacent layers equal to $30 \mathrm{meV}$ ). The potential difference induces the hybridization between MLG-like and BLG-like subbands and also shifts down in energy the top of the $\alpha$ band. $\mathbf{e}-\mathbf{h}$, The TMF spectra in TLG at $D=0$ (e), $0.18(\mathbf{f}), 0.34(\mathbf{g})$ and $0.49 \mathrm{~V} \mathrm{~nm}^{-1}(\mathbf{h})$. As $D$ increases, the $\alpha$ band starts to disappear whereas the $\beta$ band remains visibly unchanged.

into a partly triangular surface, altering therefore the carrier's trajectory and the values of $B_{\mathrm{f}}$ (see Supplementary Information).

Even though TMF cannot be used to differentiate MLG from BLG, the TMF spectrum of TLG is remarkably different because of the multiband character of its band structure. Figure $2 \mathrm{c}$ shows the TMF fan of TLG, measured at zero electric displacement field. The band structure of TLG consists of a massless MLG-like and a massive BLG-like subband at low energy ${ }^{9,16-19}$ (Fig. 2c, inset). Both subbands give rise to their own TMF spectra, with the BLG-like subband having a larger $B_{\mathrm{f}}^{(1)}$ owing to its larger Fermi momentum (for a given $E_{\mathrm{F}}$ ). This allows us to identify the subband corresponding to each peak observed in the data. At high density, the peak from the MLG-like subband can be seen at $\sim 250 \mathrm{mT}$ (Fig. 2c, orange dashed line) whereas the peaks from the BLG-like subband are visible from $\sim 250 \mathrm{mT}$ onward (Fig. 2 c, blue dashed lines). We do not observe higher-order peaks from the MLG-like subband, probably masked by the much stronger peaks from the BLG-like subband, which contains most of the charge density.

Earlier studies have shown that higher-order hopping parameters in TLG significantly modify its band structure ${ }^{9,16-19}$ by introducing subband overlap and trigonal warping in the BLG-like subband (Fig. 3a). We use this full parameter model for the TLG band structure to simulate the carrier trajectories and determine $B_{\mathrm{f}}$ (see Supplementary Information). The results are shown as dashed lines in Fig. 2c. Although we can reproduce the focusing field for the MLG-like subband very accurately, we obtain a mismatch in the BLG-like subband, similar to those mentioned above for MLG and BLG.

We now focus on the previously unexplored potential of TMF as a ballistic electron spectroscopy method to investigate in situ changes in the band structure of a material. The TLG band structure can be tuned and controlled by using a transverse electric displacement field ${ }^{20}, D$. TMF is sensitive to the occupation of each of the TLG subbands, enabling us to use TMF as a probe of the change in the TLG band structure with $D$. Figure $3 a, b$ shows the band structure and Fermi surface of TLG at $n=-2 \times 10^{12} \mathrm{~cm}^{-2}$ and $D=0$. We denote the valence bands of the MLG-like and BLG-like subbands as $\alpha$ and $\beta$ bands, respectively. A finite $D$ induces a potential difference between the layers, causing a hybridization between the MLG-like and BLG-like subbands and a shift down of the top of the $\alpha$ band (Fig. 3c,d). Consequently, for a fixed density, $k_{\mathrm{F}}$ for the $\alpha$ band shrinks with $D$ whereas that of the $\beta$ band barely changes owing to its much higher density of states.

Figure $3 \mathrm{e}-\mathrm{h}$ shows the TMF spectra of TLG at various $D$ values. We observe a relatively strong focusing peak from the $\alpha$ band at $D=0 \mathrm{~V} \mathrm{~nm}^{-1}$. However, as $D$ increases, the peak starts to shift downward and it eventually disappears at low density. The disappearance of this peak is the result of the top of the $\alpha$ band shifting down in energy and leaving the $\beta$ band as a lone contributor to the carrier density (Fig. $3 \mathrm{c}, \mathrm{d}$ ). Therefore, within our density range, a single focusing peak, from the $\beta$ band, is present at high $D$. The density onset of the $\alpha$-band focusing peak allows us to determine the potential difference among the TLG layers as a function of $D$. This allows us to estimate the effective dielectric constant of TLG $\epsilon_{\mathrm{TLG}}=3.5 \pm 0.2$ (see Supplementary Information).

We now look at the temperature dependence of the TMF spectra in MLG and BLG. The TMF spectrum is affected by temperature, $T$, at least in two ways: through the increase in dephasing (which smooths the quantum interference fluctuations), and through the loss of ballistic transport due to new scattering channels activated at high $T$. Figure $4 \mathrm{a}, \mathrm{b}$ shows the TMF spectra of MLG and BLG, respectively, at $n=-2.8 \times 10^{12} \mathrm{~cm}^{-2}$ from 0.3 to $150 \mathrm{~K}$. We first concentrate on the fine structure observed at low $T$ (blue traces). This structure is the aforementioned quantum interference between different paths on which electrons propagate to the collector ${ }^{4,11-13}$. When the temperature-induced broadening of $k_{\mathrm{F}}$ is of the order of $1 / L$, electrons become incoherent and the quantum interference is washed out ${ }^{21}$, resulting in smooth focusing peaks. For our devices, this length corresponds to $T \sim 15 \mathrm{~K}$, in good agreement with the data. 

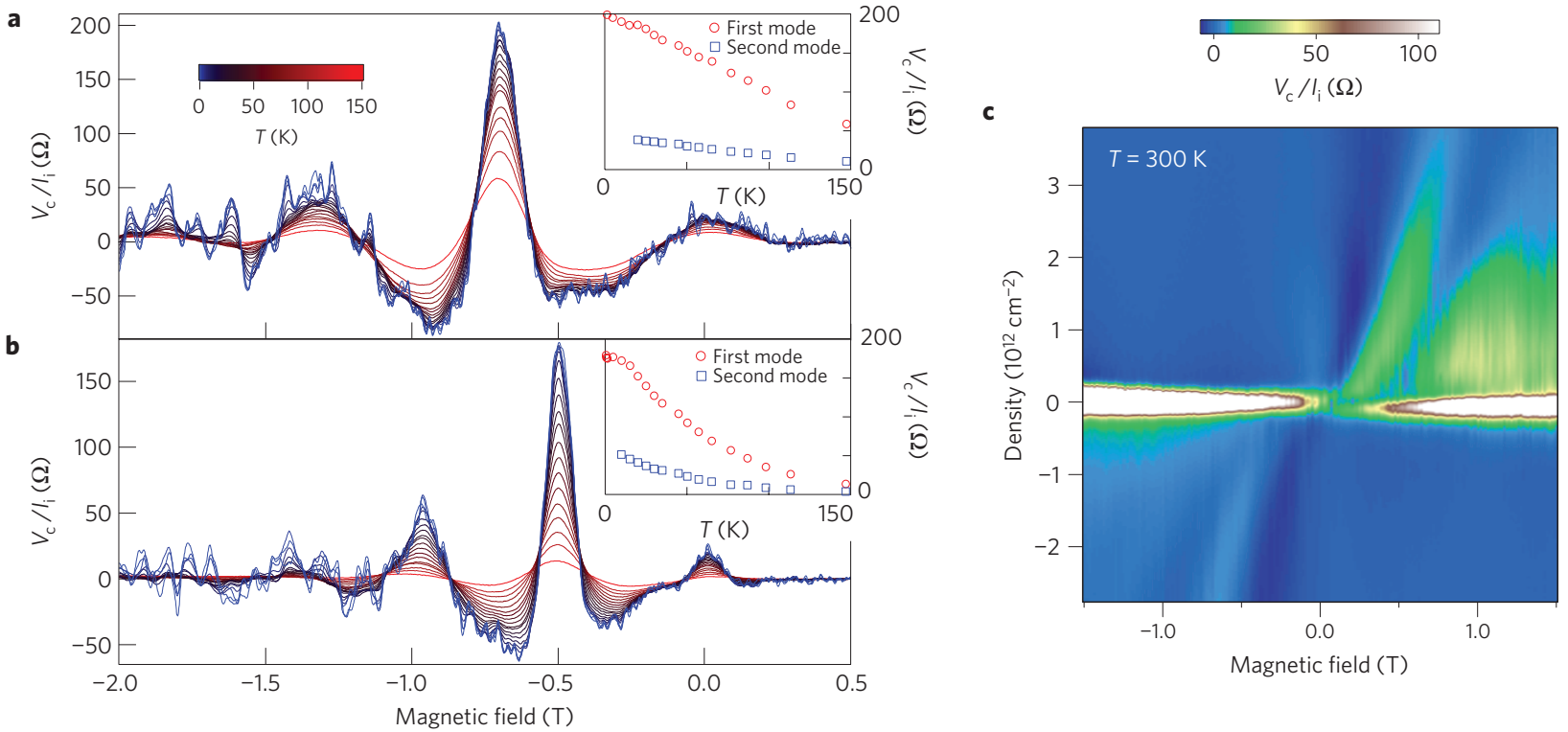

Figure 4 | Temperature dependence of the TMF in MLG and BLG. a,b, The TMF spectra as a function of temperature from $300 \mathrm{mK}$ to $150 \mathrm{~K}$ for MLG and $B L G$, respectively, at $n=-2.8 \times 10^{12} \mathrm{~cm}^{-2}$. Insets show the amplitudes of the first and second modes as a function of temperature (data taken before current annealing). c, TMF in MLG at $300 \mathrm{~K}$. The TMF peak as well as the $\sqrt{n}$ dependence of the focusing field can be clearly observed (data taken after current annealing).

In addition, the focusing peaks also decrease as $T$ increases (Fig. 4a,b, insets). We observe that the focusing amplitude in MLG depends linearly on $T$. A potential scattering mechanism includes longitudinal acoustic phonons, which give rise to a linear temperature dependence of the scattering rate ${ }^{22}$. However, we observe a very different temperature dependence in BLG. The peak amplitude saturates at low temperature and decreases faster than in MLG at higher T. A similar temperature dependence of the focusing peaks has also been observed in InGaAs/InP heterojunction ${ }^{23}$. Further theoretical work is needed to understand the temperature dependence of the focusing peaks as well as the difference between MLG and BLG.

We end by commenting on the remarkable robustness of TMF in graphene. Figure 4c shows the TMF fan of MLG at room temperature $(T=300 \mathrm{~K})$, where the first mode is clearly visible, indicating room-temperature ballistic transport well into the micrometre regime. This lower-bound temperature for the observation of TMF in graphene is at least three times higher than the highest temperature at which TMF has been observed in semiconductor heterostructures ${ }^{23}$, the main reason probably being the lack of remote interfacial phonon scattering ${ }^{24}$ from $\mathrm{hBN}$. The ability to manipulate ballistic motion in graphene at room temperature, coupled with recent developments ${ }^{25}$ in large-area growth of graphene on hBN, paves the way towards new applications based on electron-optics. On a more fundamental level, TMF may serve as a probe of electron-electron interaction $^{26-28}$ or strain-induced gauge field ${ }^{29-31}$ effects in the electronic structure of graphene.

\section{Methods}

Figure 1c shows an AFM image from one of our devices. Our devices are fabricated by transferring graphene onto high-quality hBN (ref. 8). We use oxygen plasma to etch graphene flakes into a Hall-bar geometry. Contacts are defined by electron-beam lithography and thermal evaporation of chromium and gold. The devices are then heat annealed in forming gas and subsequently current annealed in vacuum at low temperature ${ }^{9}$. We observe TMF peaks both before and after current annealing. The data after current annealing have higher quality than before current annealing, especially at low density, probably owing to reduced charge density fluctuations. However, they exhibit similar quality at high density. All of the data shown here are measured after current annealing, except the temperature dependence of the TMF peaks at high density (Fig. 4a,b), which was done before current annealing.

To probe TMF in graphene, we employ the measurement configuration shown in Fig. 1c. Current $I_{\mathrm{i}}$ is injected through contact $\mathrm{i}$ while contact $\mathrm{g}$ is grounded and voltage $V_{\mathrm{c}}$ is measured at the collector (contact c) relative to contact f. The magnetic field is applied normal to graphene. The measurement set-up is topologically equivalent to a longitudinal resistance measurement.

We identify the number of graphene layers by Raman spectroscopy and/or quantum Hall measurements. For TLG, the quantum Hall measurements reveal that it is Bernal-stacked ${ }^{9}$. In addition, we put a top gate onto the TLG device, using $\mathrm{hBN}$ as a thin dielectric. The combination of top gate (TG) and bottom gate (BG) allows us to control the charge density and the displacement field independently. We parameterize the displacement field by $D=\left(C_{\mathrm{TG}} V_{\mathrm{TG}}+C_{\mathrm{BG}} V_{\mathrm{BG}}\right) /\left(2 \epsilon_{0}\right)$, where $C$ is the capacitive coupling, $V$ is the applied gate voltage relative to the charge neutrality point and $\epsilon_{0}$ is the vacuum permittivity.

Received 26 October 2012; accepted 10 January 2013; published online 17 February 2013

\section{References}

1. Bloch, F. Über die Quantenmechanik der Elektronen in Kristallgittern. Z. Phys. 52, 555-600 (1929).

2. Tsoi, V. S. Focusing of electrons in a metal by a transverse magnetic field. JETP Lett. 19, 70-71 (1974).

3. Tsoi, V. S., Bass, J. \& Wyder, P. Studying conduction-electron/interface interactions using transverse electron focusing. Rev. Mod. Phys. 71, 1641-1693 (1999).

4. Van Houten, H. et al. Coherent electron focusing with quantum point contacts in a two-dimensional electron gas. Phys. Rev. B 39, 8556-8575 (1989).

5. Rokhinson, L. P., Larkina, V., Lyanda-Geller, Y. B., Pfeiffer, L. N. \& West, K. W. Spin separation in cyclotron motion. Phys. Rev. Lett. 93, 146601 (2004).

6. Goldman, V. J., Su, B. \& Jain, J. K. Detection of composite fermions by magnetic focusing. Phys. Rev. Lett. 72, 2065-2068 (1994).

7. Smet, J. H. et al. Magnetic focusing of composite fermions through arrays of cavities. Phys. Rev. Lett. 77, 2272-2275 (1996).

8. Dean, C. R. et al. Boron nitride substrates for high-quality graphene electronics. Nature Nanotech. 5, 722-726 (2010).

9. Taychatanapat, T., Watanabe, K., Taniguchi, T. \& Jarillo-Herrero, P. Quantum Hall effect and Landau-level crossing of Dirac fermions in trilayer graphene. Nature Phys. 7, 621-625 (2011).

10. Mayorov, A. S. et al. Micrometer-scale ballistic transport in encapsulated graphene at room temperature. Nano Lett. 11, 2396-2399 (2011).

11. Beenakker, C. W. J., van Houten, H. \& van Wees, B. J. Mode interference effect in coherent electron focusing. Europhys. Lett. 4, 359-364 (1988).

12. Aidala, K. E. et al. Imaging magnetic focusing of coherent electron waves. Nature Phys. 3, 464-468 (2007). 
13. Rakyta, P., Kormányos, A., Cserti, J. \& Koskinen, P. Exploring the graphene edges with coherent electron focusing. Phys. Rev. B 81, 115411 (2010).

14. Silvestrov, P. G. \& Efetov, K. B. Charge accumulation at the boundaries of a graphene strip induced by a gate voltage: Electrostatic approach. Phys. Rev. B 77, 155436 (2008).

15. McCann, E. \& Fal'ko, V. I. Landau-level degeneracy and quantum Hall effect in a graphite bilayer. Phys. Rev. Lett. 96, 086805 (2006).

16. Lu, C. L., Chang, C. P., Huang, Y. C., Chen, R. B. \& Lin, M. L. Influence of an electric field on the optical properties of few-layer graphene with $\mathrm{AB}$ stacking. Phys. Rev. B 73, 144427 (2006).

17. Guinea, F., Neto, A. H. C. \& Peres, N. M. R. Electronic states and Landau levels in graphene stacks. Phys. Rev. B 73, 245426 (2006).

18. Latil, S. \& Henrard, L. Charge carriers in few-layer graphene films. Phys. Rev. Lett. 97, 036803 (2006).

19. Partoens, B. \& Peeters, F. M. From graphene to graphite: Electronic structure around the K point. Phys. Rev. B 74, 075404 (2006).

20. Koshino, M. \& McCann, E. Gate-induced interlayer asymmetry in ABA-stacked trilayer graphene. Phys. Rev. B 79, 125443 (2009).

21. Cheianov, V. V., Falko, V. \& Altshuler, B. L. The focusing of electron flow and a veselago lens in graphene $p-n$ junctions. Science 315, 1252-1255 (2007).

22. Hwang, E. H. \& Das Sarma, S. Acoustic phonon scattering limited carrier mobility in two-dimensional extrinsic graphene. Phys. Rev. B 77, 115449 (2008).

23. Heremans, J., Fuller, B. K., Thrush, C. M. \& Partin, D. L. Temperature dependence of electron focusing in $\operatorname{In}_{1-x} \mathrm{Ga}_{x} \mathrm{As} / \mathrm{InP}$ heterojunctions. Phys. Rev. B 52, 5767-5772 (1995).

24. Schiefele, J., Sols, F. \& Guinea, F. Temperature dependence of the conductivity of graphene on boron nitride. Phys. Rev. B 85, 195420 (2012).

25. Liu, Z. et al. Direct growth of graphene/hexagonal boron nitride stacked layers. Nano Lett. 11, 2032-2037 (2011).

26. Mayorov, A. S. et al. Interaction-drive spectrum reconstruction in bilayer graphene. Science 333, 860-863 (2011).

27. Weitz, R. T., Allen, M. T., Feldman, B. E., Martin, J. \& Yacoby, A. Broken-symmetry states in doubly gated suspended bilayer graphene. Science 330, 812-816 (2010).
28. Kotov, V. N., Uchoa, B., Pereira, V. M., Guinea, F. \& Castro Neto, A. H. Electron-electron interactions in graphene: Current status and perspectives. Rev. Mod. Phys. 84, 1067-1125 (2012).

29. Guinea, F., Katsnelson, M. I. \& Geim, A. K. Energy gaps and a zero-field quantum Hall effect in graphene by strain engineering. Nature Phys. 6, 30-33 (2010).

30. Levy, N. et al. Strain-induced pseudo-magnetic fields greater than 300 tesla in graphene nanobubbles. Science 329, 544-547 (2010),

31. Gomes, K. K., Mar, W., Ko, W., Guinea, F. \& Manoharan, H. C. Designer Dirac fermions and topological phases in molecular graphene. Nature $\mathbf{4 8 3}$, 306-310 (2012).

\section{Acknowledgements}

We thank L. Levitov and A. Yacoby for discussions. We acknowledge financial support from National Science Foundation Career Award No. DMR-0845287 and the Office of Naval Research GATE MURI. This work made use of the MRSEC Shared Experimental Facilities supported by the National Science Foundation under award No. DMR-0819762 and of Harvard's Center for Nanoscale Systems (CNS), supported by the National Science Foundation under grant No. ECS-0335765.

\section{Author contributions}

T. Taychatanapat fabricated the samples and performed the experiments. K.W. and T. Taniguchi synthesized the hBN samples. T. Taychatanapat and P.J-H. carried out the data analysis and co-wrote the paper.

\section{Additional information}

Supplementary information is available in the online version of the paper. Reprints and permissions information is available online at www.nature.com/reprints. Correspondence and requests for materials should be addressed to P.J-H.

\section{Competing financial interests}

The authors declare no competing financial interests. 